\title{
Conservative Approach Might Be the Strategy by Default in Stable Angina Patients: A Case Report
}

\section{Serigne Cheikh Tidiane Ndao, Mame Madjiguene Ka, Djibril Marie Ba, Khadidiatou Dia, Mouhamed Chérif Mboup, Pape Diadie Fall}

Department of Cardiology, Hospital Principal Dakar, Dakar, Senegal

Email: sctndao@gmail.com

How to cite this paper: Ndao, S.C.T., Ka, M.M., Ba, D.M., Dia, K., Mboup, M.C. and Fall, P.D. (2020) Conservative Approach Might Be the Strategy by Default in Stable Angina Patients: A Case Report. World Journal of Cardiovascular Diseases, 10, 769-774. https://doi.org/10.4236/wjcd.2020.1011073

Received: September 22, 2020 Accepted: November 22, 2020 Published: November 25, 2020

\section{Copyright $\odot 2020$ by author(s) and} Scientific Research Publishing Inc. This work is licensed under the Creative Commons Attribution International License (CC BY 4.0).

http://creativecommons.org/licenses/by/4.0/ (c) (i) Open Access

\begin{abstract}
Management of stable angina is still a matter of debate. Whether a conservative or invasive approach is better remains unclear. Even though recent large-scale randomized trials depict the conservative strategy as safe and efficient. In this report, we present a case of a 53-year-old male patient with cardiovascular risk factors of hypertension and hypercholesterolemia. He complained about typical chest pain at exertion. The coronary angiogram (CA) revealed severe stenosis of the proximal left anterior descending artery (LAD) ostium. After heart team discussion, coronary artery bypass graft (CABG) was advocated. However, due to a lack of means, the CABG was postponed. Meanwhile, he was managed with guideline-based medical therapy. In addition, he exercised regularly and adopted a healthy diet. Evolution was favorable with excellent symptoms control. A regular follow-up was organized with his cardiologist. A new $C A$ before an eventual CABG six years later showed a significant plaque volume regression of the proximal LAD. We decided to continue conservative management. The stress echocardiogram to assess symptoms and exercise tolerance was normal. Our patient was sent to surgery on top of optimal medical therapy even though angioplasty was a good indication and was feasible. However, while he was struggling to afford the CABG procedure, he adopted a very healthy lifestyle along with medical therapy. Six years later, the result of that approach was without appeal, suggesting and reinforcing the conservative management of stable heart disease over invasive strategy. The recent ISCHEMIA trial is a major argument supporting that approach.
\end{abstract}

\section{Keywords}

Stable Angina, Chronic Coronary Syndrome, Conservative Treatment, Case Report 


\section{Background}

Stable ischemic heart disease (SIHD) remained the leading cause of death in many countries [1]. It's revealed classically by angina but may have various clinical presentations. It represents one of the numerous scenarios of the chronic coronary syndrome as defined by the recent ESC guideline [2]. It's a growing matter of interest since its management is not well established. In fact, we still, do not clearly know the best approach between conservative and invasive management in these patients.

Even though, important data from clinical randomized trials demonstrated that these two strategies make equal play [3] [4].

We report a case of stable angina patient related to a severe left anterior descending artery (LAD) stenosis managed medically with striking evolution.

\section{Case Presentation}

A 53-year-old man with unremarkable past medical history and cardiovascular risk factors of hypertension and hypercholesterolemia presented with stable angina.

History began with a constrictive chest pain associated with dyspnea occurring during walking with rapid progression leading the patient to consult his cardiologist.

During initial workup:

- His physical examination was normal;

- Laboratory data was unremarkable except for an abnormal lipid profile: low-density-lipoprotein cholesterol (LDL) $1.41 \mathrm{~g} / \mathrm{l}$; total cholesterol $2.11 \mathrm{~g} / \mathrm{l}$, high-density-lipoprotein cholesterol (HDL) $0.68 \mathrm{~g} / \mathrm{l}$, triglycerides $0.61 \mathrm{~g} / \mathrm{l}$, of note ultrasensitive troponin was within normal range;

- Twelve-lead ECG during the initial presentation showed negative $\mathrm{T}$ waves in the anterior leads (Figure 1);

- An echocardiogram demonstrated a moderate hypokinesis of the septo-apical wall with a normal left ventricular ejection fraction (LVEF) of 61\% Simpson biplane.

Due to the typical chest pain, the patient underwent a coronary angiogram without a prior stress test. It showed severe stenosis (90\%) of the proximal left anterior descending artery (LAD) ostium (Figure 2(A1), Figure 2(B1)). Of note, intracoronary nitrates administration didn't improve the degree of the stenosis. The other coronary arteries were normal.

After the heart team discussion, the patient was sent to surgery for coronary artery bypass graft (CABG). Meanwhile optimal medical treatment comprising ATENOLOL $100 \mathrm{mg}$ od; ATORVASTATIN $80 \mathrm{mg}$ od; ASPIRIN $100 \mathrm{mg}$ od; TRIMETAZIDINE $35 \mathrm{mg}$ bid; MOLSIDOMINE $2 \mathrm{mg}$ bid, AMLODIPINE $10 \mathrm{mg}$ od was prescribed. However, due to financial concerns, the patient didn't undergo surgery.

He adopted a very healthy lifestyle. He exercised regularly (around 45 minutes 
of running at least 5 days a week) along with a healthy diet (close as much as the Mediterranean regimen) and full adherence to his treatment and follow-up while struggling to afford CABG procedure.

Six years later, a control coronary angiogram before an eventual CABG revealed an astonishing recovery of the ostial LAD stenosis (Figure 2(A2), Figure 2(B2)).

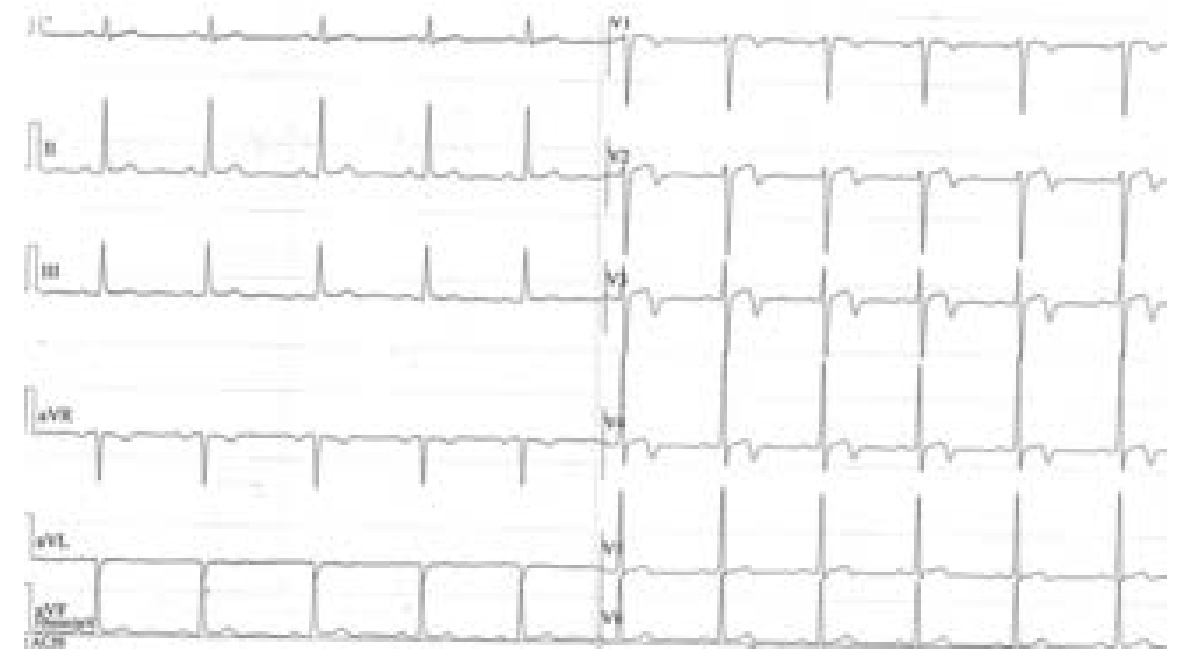

Figure 1. First ECG showing sinus rhythm with negative T wave in the anterior leads.

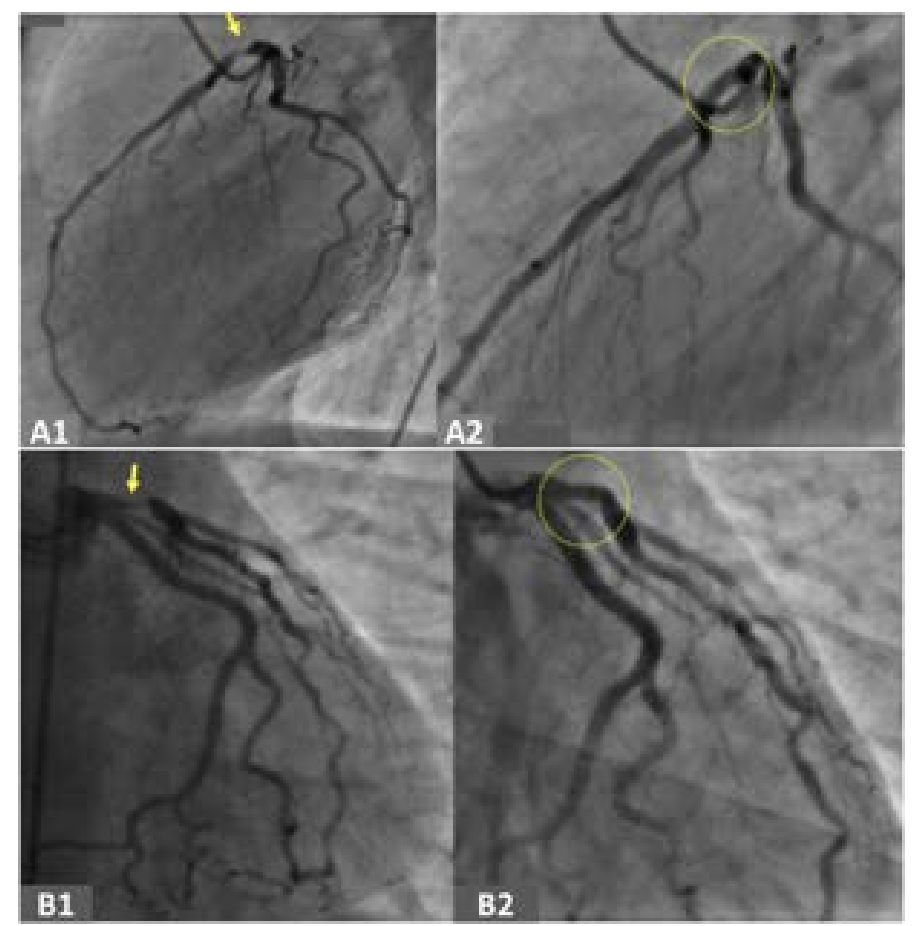

Figure 2. Coronary angiograms. Initial coronary angiogram with very tight stenosis of the ostium of the proximal left anterior descending coronary artery, in left lateral view (A1) and antero-posterior 0 - caudal $30^{\circ}$ (B1) views, see yellow arrow. Control coronary angiogram with significant regression of the ostial LAD plaque in respective views in (A2) and (B2), see yellow broken line circle. 
- Laboratory data showed a better lipid profile (LDL 0.94 g/l; CT 1.54 g/l; HDL $0.57 \mathrm{~g} / \mathrm{l}$; TGD $0.77 \mathrm{~g} / \mathrm{l})$;

- Twelve-lead ECG, before coronary angiogram, was normal (Figure 3);

- The echocardiogram showed no evidence of structural heart disease, LVEF was normal ( $65 \%$ by Simpson Biplane);

- We decided to keep the patient on conservative guideline-based medical therapy after exercise stress echocardiogram assessment;

- The latter, performed under optimal treatment (ATENOLOL 100 MG daily, was normal. The patient reached $95 \%$ of workload predicted for age and sex and 150 Watts without chest pain and wall motion abnormalities.

We recommended to the patient to continue healthy lifestyle and medical observance through regular follow-up with his cardiologist.

\section{Discussion}

Stable angina is included in the chronic coronary syndrome (CCS) new entity since the latest guidelines of the European Society of cardiology on the subject [2]. It is a very dynamic area in terms of management and still a matter of controversy.

Our patient's presentation was rapidly progressive stable angina, leading his cardiologist to send him directly to the catheterization laboratory for coronary angiography. One may skip functional ischemic testing when symptoms are very typical as recommended by the latest ESC guideline [2].

The patient was sent to surgery after a heart team discussion given his young age and the anatomic particularities of the lesion, characterized by a trifurcation lesion and ostial proximal LAD lesion, which can also be described as left main trifurcation lesion with one vessel involvement. However, he was also suitable for angioplasty, his Syntax score was 13. According to the recent ESC guideline on the revascularization, proximal LAD stenosis (or left main disease with low SYNTAX score $(0-22)$ can be addressed by angioplasty as well as by CABG with IA class of recommendation [5].

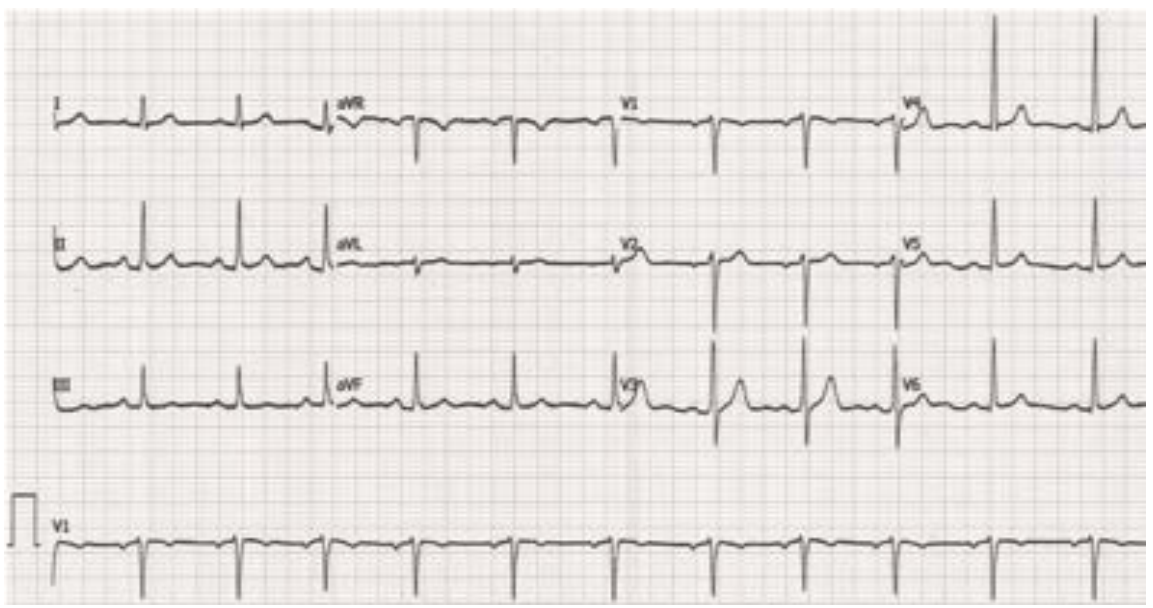

Figure 3. ECG2 showing sinus rhythm with normal repolarization pattern. 
CABG wasn't performed, but fortunately, the patient was a stickler for his treatment and lifestyle modification, as witnessed by the significant reduction of the LDL cholesterol. Of note, he was naive of antianginal drugs and disease-modifying agents before the coronary angiogram. He exercised regularly and had a consistent follow-up. Thus, the regression of the ostial LAD plaque is the fruit of a well-conducted guideline-based medical therapy and a healthy lifestyle. The main consequence of these measures being the intensive plasma lipid reduction, appears to have a major impact on plaque volume regression as demonstrated by large-scale clinical trials [6] [7].

Mechanisms by which lipid-lowering may reduce and stabilize plaque volume have been well documented. A recent pooled analysis of several serial coronary IVUS trials provided strong insights as to how statins may stabilize plaque beyond their effects on plaque regression [8]. These results demonstrate that statins, particularly high-intensity statin therapy, stabilize plaque by promoting calcification in addition to their plaque regressing-effects. Furthermore, no need to mention the clinical benefit associated with those effects [9] [10].

Thus, our case report adds credit to conservative strategy recommended in patients with stable angina. A similar case with the multivessel disease showed excellent symptoms control and an increase in exercise tolerance with optimal medical therapy alone [11].

The International Study of Comparative Health Effectiveness with Medical and Invasive Approaches (ISCHEMIA) demonstrated no significant difference between the two strategies in the rate of death from cardiovascular causes, myocardial infarction, or hospitalization for unstable angina, heart failure, or resuscitated cardiac arrest [3]. This recent randomized clinical trial, as well as the previous one like COURAGE4, shows us that wisdom lays on a conservative approach in stable patients, at least as the first intention. However, one should keep in mind, as clearly stated by the guidelines, that revascularization should be considered in case of persisting angina despite optimized medical therapy. Furthermore, we should consider the "PCI-first approach" to improve prognosis particularly in patients with LM disease $>50 \%$, multivessel disease, in the presence of LV dysfunction, or large ( $>10 \% \mathrm{LV})$ area of ischemia.

\section{Conclusion}

Stable angina, even severe, can be managed efficiently by medical therapy along with healthy lifestyle modification. There is growing evidence supporting a conservative strategy with guideline-based medical therapy as the approach by default in patients with stable angina.

\section{Consent}

The authors confirm that written consent for submission and publication of the image(s) and associated text has been obtained from the patient (or next of kin where appropriate) in line with COPE guidance. 


\section{Conflicts of Interest}

The authors declare no conflicts of interest regarding the publication of this paper.

\section{References}

[1] Benjamin, E.J., Virani, S.S., Callaway, C.W., et al. (2018) American Heart Association Council on Epidemiology and Prevention Statistics Committee and Stroke Statistics Subcommittee. Heart Disease and Stroke Statistics-2018 Update: A Report from the American Heart Association. Circulation, 137, :e67-e492. https://doi.org/10.1161/CIR.0000000000000573

[2] Knuuti, J., Wijns, W., Saraste, A., Capodanno, D., Barbato, E., Funck-Brentano, C., et al. (2020) 2019 ESC Guidelines for the Diagnosis and Management of Chronic Coronary Syndromes. European Heart Journal, 41, 407-477.

[3] Maron, D.J., Hochman, J.S., Reynolds, H.R., Bangalore, S., O’Brien, S.M., Boden, W.E., et al. (2020) Initial Invasive or Conservative Strategy for Stable Coronary Disease. New England Journal of Medicine, 382, 1395-1407.

[4] Boden, W.E., O'Rourke, R.A., Teo, K.K., Hartigan, P.M., Maron, D.J., Kostuk, W.J., et al. (2007) Optimal Medical Therapy with or without PCI for Stable Coronary Disease. New England Journal of Medicine, 356, 1503-1516. https://doi.org/10.1056/NEJMoa070829

[5] Neumann, F.J., Sousa-Uva, M., Ahlsson, A., Alfonso, F., Banning, A.P., Benedetto, U., et al. (2019) 2018 ESC/EACTS Guidelines on Myocardial Revascularization. European Heart Journal, 40, 87-165. https://doi.org/10.1093/eurheartj/ehy855

[6] Chhatriwalla, A.F., Nicholls, S.J., Wang, T.H., Wolski, K., Sipahi, I., Crowe, T., et al. (2009) Low Levels of Low-Density Lipoprotein Cholesterol and Blood Pressure and Progression of Coronary Atherosclerosi. Journal of the American College of Cardiology, 53, 1110-1115. https://doi.org/10.1016/j.jacc.2008.09.065

[7] Corti, R., Fuster, V., Fayad, Z.A., Worthley, S.G., Helft, G., Chaplin, W.F., et al. (2005) Effects of Aggressive versus Conventional Lipid-Lowering Therapy by Simvastatin on Human Atherosclerotic Lesions. A Prospective, Randomized, Double-Blind Trial with High-Resolution Magnetic Resonance Imaging. Journal of the American College of Cardiology, 46, 106-112. https://doi.org/10.1016/j.jacc.2005.03.054

[8] Puri, R., Nicholls, S.J., Shao, M., Kataoka, Y., Uno, K., Kapadia, S.R., et al. (2015) Impact of Statins on Serial Coronary Calcification during Atheroma Progression and Regression. Journal of the American College of Cardiology, 65, 1273-1282. https://doi.org/10.1016/j.jacc.2015.01.036

[9] Mozaffarian, D., Benjamin, E.J., Go, A.S., Arnett, D.K., Blaha, M.J., Cushman, M., et al. (2015) Heart Disease and Stroke Statistics-2015 Update: A Report from the American Heart Association. Circulation, 131, e29-e322.

[10] Cholesterol Treatment Trialists (CTT) Collaboration (2010) Efficacy and Safety of More Intensive Lowering of LDL Cholesterol: A Meta-Analysis of Data from 170000 Participants in 26 Randomised Trials. Lancet, 376, 1670-1681. https://doi.org/10.1016/S0140-6736(10)61350-5

[11] Gowdak, L.H.W. (2019) A Patient with Stable Angina and Mild Ischemia: Do I Have the COURAGE Not to Stent the Lesion?. Heart \& Metabolism, 78, 24-27. 